\title{
Repair of damaged pancreatic $\beta$ cells: New hope for a type 2 diabetes reversal?
}

\author{
Zhili Wang, Haiyan Xiong, Tu Ya Sa Ren \\ Beijing Chaoyang Diabetes Hospital, Beijing 100025, China
}

Type 2 diabetes mellitus (T2DM) is a complex and heterogeneous metabolic disorder characterized by the damage and eventual loss of pancreatic $\beta$ cells resulting in abnormal insulin secretion. Pancreatic $\beta$-cell dysfunction, including reduction of $\beta$-cell mass and function, plays key role in the onset and progression of T2DM. ${ }^{[1]}$ Influenced by the findings of the UK Prospective Diabetes Study (UKPDS), the traditional view is that once diabetes is diagnosed, $\beta$-cell function decreases at a rate of $4 \%$ per year and the conventional treatment cannot reverse its progress. ${ }^{[2]}$ This is the background to the failure in addressing the two key causes of diabetes, namely, insulin resistance and $\beta$-cell failure.

Recent pathophysiological and clinical studies have provided us with a deeper understanding of diabetes. Apoptosis has been considered as the main contributor of $\beta$-cell dysfunction and decrease in $\beta$-cell mass for a long time. However, more recent studies have shown that the loss of functional $\beta$-cell mass is not as much due to $\beta$-cell death, but rather due to $\beta$-cell dedifferentiation when the $\beta$ cells are exposed to metabolic stressors, and islet $\beta$-cell dedifferentiation may be more important than $\beta$-cell apoptosis in T2DM. ${ }^{[1,3]}$ Moreover, the dedifferentiation of islet $\beta$ cells is reversible, which provides opportunities to prevent or reverse $\beta$-cell loss in T2DM. $\cdot^{[3]}$ In the wake of injury factors and metabolic stress, $\beta$ cells become dedifferentiated to resist external injuries. They lose their differentiation characteristics and stop secreting insulin, but still express the markers of progenitor cells. If $\beta$ cells do not die or stay in the late stage of cell degeneration during the process of apoptosis, they will become dedifferentiated cells in the quiescent phase. Before irreversible changes occur, removal of the damage factors can drive $\beta$ cells to undergo redifferentiation and restore its function. ${ }^{[4]}$ More evidence proved that T2DM reversal or clinical cure is achievable by using lifestyle changes, ${ }^{[4]}$ intensive insulin therapy, ${ }^{[5]}$ bariatric surgery, low-calorie diets (LCD), or carbohydrate restriction. ${ }^{[6]}$ Shortterm intensive insulin therapy can improve the pathophysiological defects underlying T2DM (insulin resistance, pancreatic $\beta$-cell dysfunction, and $\alpha$-cell dysfunction)..$^{[5,7]}$ Taylor et al ${ }^{[8]}$ have shown that T2DM is a condition mainly caused by excessive, yet reversible, fat accumulation in the liver and pancreas, and reversal of T2DM requires a reduction in fat content in the liver and pancreas. The Diabetes Remission Clinical Trial (DiRECT) demonstrated that the ability of $\beta$ cells in recovering their long-term function persists after diagnosis, changing the previous paradigm of irreversible loss of $\beta$-cell function in T2DM. ${ }^{[8]}$ In addition, dysfunction of islet microcirculation is also involved in the pathogenesis of diabetes mellitus. ${ }^{[9]}$ Dysfunction or destruction of the islet microenvironment caused mainly by abnormal microcirculation in pancreatic islets is the potential underlying mechanism contributing to $\beta$-cell failure in diabetes. ${ }^{[10]}$ Therefore, improving the microcirculation of the pancreatic islets can increase the blood supply of islets, improve the surrounding local metabolic environment, and contribute to the repair of damaged $\beta$ cells. In summary, $\beta$-cell dedifferentiation has been shown to be an important factor in $\beta$-cell dysfunction. 
But this dedifferentiation can be reversed by reducing the metabolic overloading of $\beta$ cells and improving the local metabolic environment.

We have incorporated the concept of protecting and restoring islet cells, thus increasing $\beta$-cell functions in our extensive clinical treatment of diabetes patients. Within a short period of time on our regime, the toxicity of hyperglycemia, including damage to small and medium blood vessels and islet microcirculation, is reduced. Concurrently, the biological activity of islet cells and insulin receptors is boosted, allowing the injured islet cells to acquire different degrees of repair, restore their normal function to various degrees, and produce more and higher-quality endogenous insulin. In this way, not only can it control the blood sugar steadily, but also create a good metabolic internal environment for the repair of damaged islet cells. Under an appropriate environment, it is possible to repair the damaged islet cells, or even reverse them to normal cells.

With our systematic treatment regime to protect and repair pancreatic islet cells, a considerable number of T2DM patients in our hospital have achieved the outcome of $\beta$-cell repair or even reversals in diabetes. A recent case of a male T2DM patient with fatty liver, severe hyperglycemia, and $\beta$-cell failure was admitted to our hospital. Following a 3 -week treatment, the patient exhibited excellent glycemic control with a normal blood glucose level and restored $\beta$-cell function without further need for blood glucose medications. In addition, his fatty liver disappeared. Our regime involved using first an insulin pump (short-term insulin intensive therapy) to quickly remove the hyperglycemic state, improve the hyperglycemic and hypertonic abnormal metabolic internal environment, and provide a metabolic local environment suitable for pancreatic $\beta$-cell recuperate and repair. Next a systematic treatment to improve the pancreatic islet microcirculation was conducted to accelerate the flow rate of the microcirculation and increase the ability and effectiveness of oxygen and nutrient supply to the whole body, including pancreatic islet cells. In the meantime, the patient received normal diabetes education, which consisted of a proper diet therapy (low calorie, low carbohydrate, high dietary fiber, and high vitamin) and a regular exercise schedule. Since the patient had diabetic peripheral neuropathy, a trophic nerve therapy was given to maintain the normal excitability of nerve fibers or repair the demyelination-like changes of nerve fibers. Following the systematic treatments above, the patient was discharged with a normal blood glucose level. We are monitoring him closely to collect more follow-up data.

In summary, our systematic treatment regime can repair the damaged pancreatic islet cells to various degrees, preventing, delaying, or avoiding the occurrence and development of diabetic complications. It would contribute to improving the longevity and life quality of diabetic patients.

\section{Conflict of Interest}

None declared.

\section{REFERENCE}

1. Khin PP, Lee JH, Jun HS. A Brief Review of the Mechanisms of $\beta$-Cell Dedifferentiation in Type 2 Diabetes. Nutrients 2021;13(5):1593

2. Matthews DR, Cull CA, Stratton IM, Holman RR, Turner RC. UKPDS 26: Sulphonylurea failure in non-insulin-dependent diabetic patients over six years. UK Prospective Diabetes Study (UKPDS) Group. Diabet Med 1998;15:297-303.

3. Accili D. Insulin Action Research and the Future of Diabetes Treatment: The 2017 Banting Medal for Scientific Achievement Lecture. Diabetes 2018;67:1701-9.

4. Gong Q, Zhang P, Wang J, Ma J, An Y, Chen Y, et al. Da Qing Diabetes Prevention Study Group. Morbidity and mortality after lifestyle intervention for people with impaired glucose tolerance: 30 -year results of the Da Qing Diabetes Prevention Outcome Study. Lancet Diabetes Endocrinol 2019;7:452-61.

5. Kramer CK, Zinman B, Retnakaran R. Short-term intensive insulin therapy in type 2 diabetes mellitus: a systematic review and meta-analysis. Lancet Diabetes Endocrinol 2013;1:28-34.

6. Hallberg SJ, Gershuni VM, Hazbun TL, Athinarayanan SJ. Reversing Type 2 Diabetes: A Narrative Review of the Evidence. Nutrients 2019;11:766.

7. Zheng HL, Xing Y, Li F, Ding W, Ye SD. Effect of short-term intensive insulin therapy on a-cell function in patients with newly diagnosed type 2 diabetes. Medicine (Baltimore) 2020;99:e19685.

8. Taylor R, Al-Mrabeh A, Sattar N. Understanding the mechanisms of reversal of type 2 diabetes. Lancet Diabetes Endocrinol 2019;7:726-36.

9. Li X, Zhang L, Meshinchi S, Dias-Leme C, Raffin D, Johnson JD, et al. Islet microvasculature in islet hyperplasia and failure in a model of type 2 diabetes. Diabetes 2006;55:2965-73.

10. Almaça J, Caicedo A, Landsman L. Beta cell dysfunction in diabetes: the islet microenvironment as an unusual suspect. Diabetologia 2020;63:2076-85.

How to cite this article: Wang $\mathrm{Z}$, Xiong $\mathrm{H}$, Ren TYS. Repair of damaged pancreatic $\beta$ cells: New hope for a type 2 diabetes reversal?. J Transl Intern Med 2021; 9: 150-1. 